\title{
Derivation modules of orthogonal duals of hyperplane arrangements
}

\author{
Joseph P. S. Kung • Hal Schenck
}

Received: 1 November 2005 / Accepted: 27 January 2006 / Published online: 11 July 2006

(C) Springer Science + Business Media, LLC 2006

\begin{abstract}
Let $A$ be an $n \times d$ matrix having full rank $n$. An orthogonal dual $A^{\perp}$ of $A$ is a $(d-n) \times d$ matrix of rank $(d-n)$ such that every row of $A^{\perp}$ is orthogonal (under the usual dot product) to every row of $A$. We define the orthogonal dual for arrangements by identifying an essential (central) arrangement of $d$ hyperplanes in $n$-dimensional space with the $n \times d$ matrix of coefficients of the homogeneous linear forms for which the hyperplanes are kernels. When $n \geq 5$, we show that if the matroid (or the lattice of intersection) of an $n$-dimensional essential arrangement $\mathcal{A}$ contains a modular copoint whose complement spans, then the derivation module of the orthogonally dual arrangement $\mathcal{A}^{\perp}$ has projective dimension at least $\lceil n(n+2) / 4\rceil-3$.
\end{abstract}

Keywords Hyperplane arrangement - Module of derivations · Projective dimension · Matroid · Orthogonal duality

\section{Introduction}

An important conjecture in the theory of hyperplane arrangements is Terao's conjecture [14]: whether the derivation module $D(\mathcal{A})$ of a central arrangement $\mathcal{A}$ is free depends only on the "combinatorics," that is to say, the matroid of $\mathcal{A}$. Since being free is equivalent to having zero projective dimension, a natural extension of Terao's conjecture is that the projective dimension $\operatorname{pdim}(D(\mathcal{A}))$ of $D(\mathcal{A})$ depends

Hal Schenck partially supported by NSF DMS 03-11142, NSA MDA 904-03-1-0006, and ATP 010366-0103.

J. P. S. Kung

Department of Mathematics, University of North Texas, Denton, TX 76203

e-mail: kung@unt.edu

H. Schenck $(\triangle)$

Department of Mathematics, Texas A\&M University, College Station, TX 77843

e-mail: schenck@math.tamu.edu 
only on the matroid of $\mathcal{A}$. No counterexamples to this extended conjecture are known, although in [21] Ziegler gives two arrangements with the same matroid but nonisomorphic derivation modules. The common matroid in Ziegler's example has rank 3 and is the truncation of the orthogonal dual $M^{\perp}\left(K_{3,3}\right)$ of the cycle matroid of the complete bipartite graph $K_{3,3}$. This matroid has two inequivalent representations, one from projecting a representation of $M^{\perp}\left(K_{3,3}\right)$ from a point in general position, the other, a less special one which cannot be "erected". However, the derivation modules of these two arrangements both have projective dimension 1. Ziegler's examples provide one motivation to study derivation modules of orthogonal duals of arrangements. Another motivation comes from computer experiments suggesting that derivation modules of duals of free arrangements tend to have high projective dimension.

The notion of orthogonal duality is pervasive in combinatorics. H. Whitney first defined duality for matroids in his 1932 paper [16] to extend the notion of a dual or face graph of a planar graph to arbitrary graphs. He proved the theorem (equivalent to Kuratowski's theorem for planarity) that a graph is planar if and only if its matroid dual is a graphic matroid. Another example (suggested by a referee) is the concept of association introduced by Coble in [2]; for an application to generic arrangements see [5]. Duality also occurs in linear programming, combinatorial optimization, and coding theory. It is closely related to Alexandrov and other kinds of duality in algebraic topology. See, for example, [3, 4, 10].

Let $X$ be a subspace in the lattice $L_{\mathcal{A}}$ of intersection of the arrangement $\mathcal{A}$. The closed subarrangement $\mathcal{A}_{X}$ is the subset of all hyperplanes in $\mathcal{A}$ containing $X$. When $X$ is 1-dimensional, $\mathcal{A}_{X}$ is a copoint. A closed subarrangement $\mathcal{A}_{X}$ (or its associated subspace $X$ ) is modular if

$$
\operatorname{rank}(X \vee Y)+\operatorname{rank}(X \cap Y)=\operatorname{rank}(X)+\operatorname{rank}(Y)
$$

for every subspace $Y$ in $L_{\mathcal{A}}$. Chains of modular flats occur (by definition) in supersolvable arrangements. In addition, it is easy to show by induction and the addition-deletion lemma (see [14] or [9], Chap. 4) that if an arrangement $\mathcal{A}$ has a modular copoint $\mathcal{A}_{X}$ which is free, then $\mathcal{A}$ itself is free.

A subarrangement of an essential arrangement spans if it is essential. The ceiling $\lceil x\rceil$ of a real number $x$ is the the smallest integer greater than or equal to $x$. The floor $\lfloor x\rfloor$ of $x$ is the largest integer less than or equal to $x$.

Our main result is the following theorem.

Theorem 1.1. Let $\mathcal{A}$ be an essential arrangement over an arbitrary field with a modular copoint $X$ such that its complement $\mathcal{A} \backslash \mathcal{A}_{X}$ spans. Suppose that the dimension $n$ of $\mathcal{A}$ is at least 5 . Then the projective dimension of the derivation module of the orthogonal dual $\mathcal{A}^{\perp}$ is bounded below by $\lceil n(n+2) / 4\rceil-3$.

The proof of Theorem 1.1 is combinatorial. We show that an arrangement $\mathcal{A}$ satisfying the main hypotheses in 1.1 contains a spanning subarrangement with the same matroid as the braid arrangement $A_{n+1}$. This implies that the dual $\mathcal{A}^{\perp}$ contains a closed circuit with at least $\lceil n(n+2) / 4\rceil$ hyperplanes. The proof is completed by combining a result of Terao on projective dimension of closed subarrangements with 
results of Rose and Terao, and Yuzvinsky on the projective dimension of generic arrangements.

\section{Projective dimension of $D(\mathcal{A})$ and closed subarrangements}

In this section, we discuss the two theorems from hyperplane arrangements we need. Both theorems hold over arbitrary fields.

A generic arrangement is an arrangement of at least $n+1$ hyperplanes in $n$ dimensional space for which every subset of $n$ hyperplanes is independent. In particular, matroids of generic arrangements are uniform matroids. The following theorem is due to Rose and Terao [11] and Yuzvinsky [19].

Theorem 2.1. If $\mathcal{A}$ is a generic arrangement in $\mathbb{k}^{n}$, then $\operatorname{pdim}(D(\mathcal{A}))=n-2$.

We shall also use the following theorem of Terao [15] (see also [1]).

Theorem 2.2. If $\mathcal{A}_{X}$ is a closed subarrangement of $\mathcal{A}$, then

$$
\operatorname{pdim}(D(\mathcal{A})) \geq \operatorname{pdim}\left(D\left(\mathcal{A}_{X}\right)\right)
$$

Terao's proof is unpublished. Yuzvinsky gives a proof in [20]. For the reader's convenience, we give another proof, which is a more elementary version of the proof in [20] (but requires the hypothesis that the field $\mathbb{k}$ has characteristic zero). Let $S$ be the symmetric algebra $\operatorname{Sym}\left(V^{*}\right)$ of the dual space $V^{*}$. The algebra $S$ is isomorphic to the polynomial algebra $\mathbb{k}\left[x_{1}, x_{2}, \ldots, x_{n}\right]$, where $\left\{x_{i}\right\}$ is a dual basis for $V$. Let $\mathcal{A}=\left\{H_{i}: 1 \leq i \leq d\right\}$ and $Q$ be the polynomial $Q=\prod_{i=1}^{d} l_{i}$, where for each $i, l_{i}$ is a homogeneous linear form such that the kernel $V\left(l_{i}\right)$ of $l_{i}$ is the hyperplane $H_{i}$. The derivation module $D(\mathcal{A})$ is the $S$-module of all $S$-derivations $\theta$ such that for all $i$, $\theta\left(l_{i}\right)$ is in the principal ideal $\left\langle l_{i}\right\rangle \subseteq S$. If char $\mathbb{k}=0$, this is equivalent to the single condition $\theta(Q) \in\langle Q\rangle$. The Euler derivation $\sum x_{i} \partial / \partial x_{i}$ generates a free summand $S(-1)$ of $D(\mathcal{A})$ and

$$
D(\mathcal{A})=S(-1) \oplus D_{0}(\mathcal{A})
$$

where $D_{0}(\mathcal{A})$ is the kernel of the Jacobian matrix $J_{Q}$, the $n \times 1$ matrix with $(i, 1)$-entry equal to $\partial Q / \partial x_{i}$ (see, for example, [19]). In particular, the projective dimension of $D(\mathcal{A})$ is one less than the projective dimension of the ideal $\left\langle J_{Q}\right\rangle$ generated by the entries of the matrix $J_{Q}$.

Let $X$ be a subspace in the intersection lattice of $\mathcal{A}$. Order the hyperplanes of $\mathcal{A}$ so that the closed subarrangement $\mathcal{A}_{X}$ equals $\left\{H_{1}, H_{2}, \ldots, H_{s}\right\}$. Choose coordinates so that $X$ is the subspace $V\left(x_{1}, x_{2}, \ldots, x_{k}\right)$ defined by the equations $x_{1}=0, x_{2}=$ $0, \ldots, x_{k}=0$, and hence, a hyperplane $H_{i}$ in $\mathcal{A}_{X}$ may be written as the kernel $V\left(l_{i}\right)$ with $l_{i}$ a homogeneous linear form in $\mathbb{k}\left[x_{1}, \ldots, x_{k}\right]$. 
Let $P$ be the prime ideal $\left\langle x_{1}, \ldots, x_{k}\right\rangle$ in $S$. By our choice of coordinates, if the hyperplane $V\left(l_{i}\right)$ does not contain the subspace $X$, then $l_{i}$ equals $\gamma_{i}+\delta_{i}$ where $\gamma_{i}$ is a linear form in $\mathbb{k}\left[x_{1}, \ldots, x_{k}\right], \delta_{i}$ is a linear form in $\mathbb{k}\left[x_{k+1}, \ldots, x_{n}\right]$, and $\delta_{i} \not \equiv 0$.

Write $Q=L K$, where $L=\prod_{i=1}^{s} l_{i}$ and $K=\prod_{i=s+1}^{|\mathcal{A}|} l_{i}$. Computing the $(i, 1)$-entry of $J_{Q}$ by the product rule, we have

$$
\frac{\partial Q}{\partial x_{i}}=L \frac{\partial K}{\partial x_{i}}+K \frac{\partial L}{\partial x_{i}}
$$

By our choice of coordinates, $\partial L / \partial x_{i}=0$ when $i>k$. Hence, the Jacobian matrix $J_{Q}$ simplifies to the transpose of the matrix

$$
\left[L \frac{\partial K}{\partial x_{1}}+K \frac{\partial L}{\partial x_{1}}, \ldots, L \frac{\partial K}{\partial x_{k}}+K \frac{\partial L}{\partial x_{k}}, L \frac{\partial K}{\partial x_{k+1}}, \ldots, L \frac{\partial K}{\partial x_{n}}\right]
$$

We localize at the prime ideal $P$. In the local ring $S_{P}$, every element not in $P$ is a unit. Since each $l_{i}$ with $i>s$ has the non-zero form $\delta_{i}$ in $\mathbb{k}\left[x_{k+1}, \ldots, x_{n}\right]$, the product $K$ contains at least one monomial in $\mathbb{k}\left[x_{k+1}, \ldots, x_{n}\right]$. Hence $K$ is a unit in $S_{P}$. Similarly, $\partial K / \partial x_{i}$ is nonzero for some $i \in\{s+1, \ldots, n\}$ and still contains a nonzero monomial in $\mathbb{k}\left[x_{k+1}, \ldots, x_{n}\right]$. In particular, as an element in $S_{P}, L$ equals $K^{-1} Q$ and since $Q \in\left\langle J_{Q}\right\rangle$ by Euler's identity, $L$ is in the ideal $\left\langle J_{Q}\right\rangle_{P}$ generated by the entries of $J_{Q}$ in $S_{P}$. We conclude that

$$
\left\langle J_{Q}\right\rangle_{P}=\left\langle K \frac{\partial L}{\partial x_{1}}, \ldots, K \frac{\partial L}{\partial x_{k}}, L\right\rangle_{P}
$$

Since $K$ is a unit, it can be removed. Further, we can use Euler's relation to write the last generator $L$ as a linear combination of the first $k$ generators. We thus obtain

$$
\begin{aligned}
\left\langle J_{Q}\right\rangle_{P} & =\left\langle\frac{\partial L}{\partial x_{1}}, \ldots, \frac{\partial L}{\partial x_{k}}\right\rangle_{P} \\
& =\left\langle J_{L}\right\rangle_{P} .
\end{aligned}
$$

Since localization is an exact functor (see, for example, [6]), localizing a minimal free resolution of $\left\langle J_{Q}\right\rangle$ yields a free resolution (possibly non-minimal) of $\left\langle J_{Q}\right\rangle_{P}$, which equals $\left\langle J_{L}\right\rangle_{P}$. The free resolution obtained for $\left\langle J_{L}\right\rangle_{P}$ is also a free resolution for $\left\langle J_{L}\right\rangle$ because $L$ is in $P$. We conclude that

$$
\operatorname{pdim}\left(\left\langle J_{L}\right\rangle\right) \leq \operatorname{pdim}\left(\left\langle J_{Q}\right\rangle\right)
$$

This completes the proof of Theorem 2.2.

We remark that Theorem 2.2 fails if one does not assume that the subarrangement is closed. An easy example is the braid arrangement $A_{4}$. It is free but contains three generic subarrangements of four lines, none of them closed. 
Combining the results on generic arrangements and Theorem 2.2, we obtain a simple but useful combinatorial corollary.

Corollary 2.3. Let $\mathcal{A}$ be an arrangement whose matroid contains a generic flat of rank $r$. Then $\operatorname{pdim}(D(\mathcal{A})) \geq r-2$. In particular, if the matroid of $\mathcal{A}$ contains a closed circuit of size $m$, then $\operatorname{pdim}(D(\mathcal{A})) \geq m-3$.

This corollary extends the folk-lore lemma that an arrangement cannot be free if it contains a closed subarrangement consisting of four hyperplanes in general position in 3-dimensional space.

Let $G$ be a graph (without loops or multiple edges) with vertex set $\{1,2, \ldots, n\}$ and edge set $E$. The graphic arrangement $\mathcal{A}_{G}$ is the collection $\left\{V\left(x_{i}-x_{j}\right) \mid\{i, j\} \in E\right\}$. For example, the braid arrangement $A_{n}$ is the arrangement associated to the complete graph $K_{n}$, the graph containing all possible edges. Graphic arrangements are never essential; an arrangement from a connected graph can be made essential by suppressing a variable. For example, $A_{n}$ can be made into the essential arrangement $\hat{A}_{n}$ consisting of the hyperplanes $V\left(x_{i}\right)$ and $V\left(x_{i}-x_{j}\right)$, where $1 \leq i<j \leq n-1$.

The only generic flats in graphic arrangements are closed circuits. Closed circuits correspond to induced cycles. Thus, Corollary 2.3 also extends the reverse implication of a theorem (combining results in [13] and [14]) that a graphic arrangement is free if and only if its graph is chordal, or equivalently, its graph has no induced cycles of length greater than 3. In particular, we have:

Corollary 2.4. If a graph $G$ contains an induced cycle of length $m$, then $\operatorname{pdim}\left(D\left(\mathcal{A}_{G}\right) \geq m-3\right.$.

We close this section with some illustrations of Corollary 2.4 and several related problems.

Example 2.5. Consider the graph $G$ (with 8 vertices) given by the 1-skeleton of the cube:

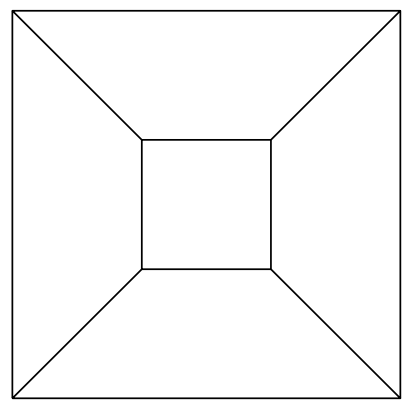


A free resolution for $D\left(\mathcal{A}_{G}\right)$ is:

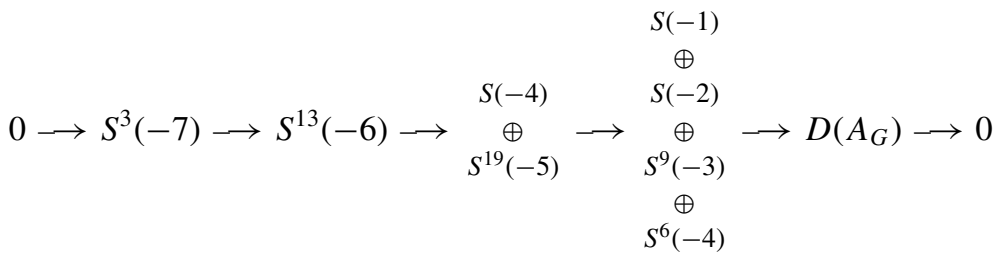

The diagram gives the degree (but not the explicit expressions) of the generators of the free modules. For example, from the diagram, one sees that $D\left(\mathcal{A}_{G}\right)$ can be generated by 17 generators, one of degree 1 (the Euler derivation), one of degree 2, nine of degree 3 , and six of degree 4 . These generators have relations which can be generated by 20 relations. The indexing of a free resolution starts at zero, and so $D\left(\mathcal{A}_{G}\right)$ has projective dimension 3 . Since $G$ has an induced cycle of length 6 , this is the lower bound predicted by Corollary 2.4 .

Example 2.6. Let $G$ be the triangular prism:

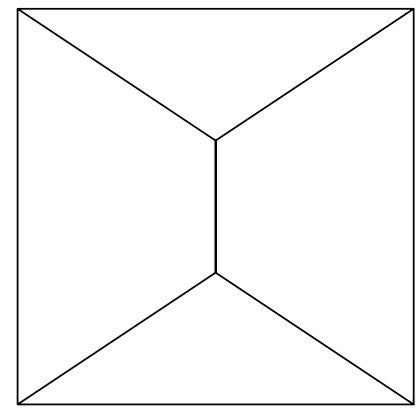

A free resolution for $D\left(\mathcal{A}_{G}\right)$ is:

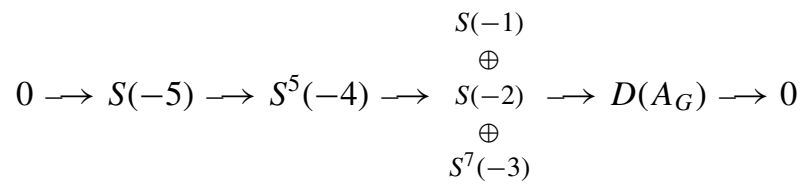

The maximum length of an induced cycle in $G$ is 4 , but the projective dimension of $D\left(\mathcal{A}_{G}\right)$ is 2 . Hence, $\operatorname{pdim}\left(D\left(\mathcal{A}_{G}\right)\right)$ can be strictly greater than the bound given in Corollary 2.4 .

Example 2.6 raises several questions. Is there a characterization of graphs $G$ for which $\operatorname{pdim}\left(D\left(\mathcal{A}_{G}\right)\right)=m-3$, where $m$ is the maximum size of an induced circuit? Are there reasonable formulas involving graph parameters for $\operatorname{pdim}\left(D\left(\mathcal{A}_{G}\right)\right)$ ? In analogy to excluded minors in matroid theory (see, for example, [8], Section 8), define an arrangement $\mathcal{A}$ to be $k$-minimal if $\operatorname{pdim}(D(\mathcal{A}))=k$ and for every proper closed subarrangement $\mathcal{A}_{X} \subset A, \operatorname{pdim}\left(D\left(\mathcal{A}_{X}\right)\right)<k$. The graphic arrangement of the triangular Springer 
prism is 2-minimal and rank- $m$ generic arrangements are $(m-2)$-minimal. It seems an interesting problem to classify $k$-minimal arrangements.

\section{Orthogonal duals of arrangements}

Let $\mathcal{A}$ be a hyperplane arrangement in $n$-dimensional space. We construct an $n \times|\mathcal{A}|$ matrix $A$ as follows: each hyperplane $H$ in $\mathcal{A}$ labels a column equal to $\left(c_{1}, c_{2}, \ldots, c_{n}\right)^{t}$, where $H=V\left(c_{1} x_{1}+c_{2} x_{2}+\cdots+c_{n} x_{n}\right)$. Conversely, given a matrix $A$, we construct an arrangement by simplifying, that is, removing all zero columns, constructing a multiset of hyperplanes corresponding to the kernels of the linear forms defined by the columns, giving a multiarrangement, and disregarding the multiplicities to obtain an arrangement.

If $\mathcal{A}$ is essential, the hyperplanes in $\mathcal{A}$ intersect in the zero subspace, and the matrix $\mathcal{A}$ has full rank $n$. The correspondence between essential arrangements $\mathcal{A}$ and $n \times|\mathcal{A}|$ matrices $A$, with no zero columns and no two columns a non-zero multiple of each other, is bijective up to left multiplication by elements of $G L(n)$, and right multiplication by a product of a permutation matrix and a non-singular diagonal matrix. The matrix $A$ is a representation for the matroid $M(\mathcal{A})$ of the arrangement $\mathcal{A}$.

Suppose that $A$ is an $n \times d$ matrix having full rank $n$. An (orthogonal) dual of $A$ is an $(d-n) \times d$ matrix $B$ having full rank $d-n$ such that any row of $A$ is orthogonal (under the usual dot product) to any row of $B$. The matrix $B$ exists and is determined up to left multiplication by a non-singular matrix. In addition, if $A^{\perp}$ is a dual of $A$, then it is also a dual of any matrix obtained from $A$ by left multiplication by a non-singular matrix. Thus, duality is an operation defined between equivalence classes of matrices. In particular, there is an easy way to construct a dual of $A$. Put $A$ into the form $[I \mid C]$, where $I$ is the $n \times n$ identity matrix. Then a dual of $A$ is $\left[-C^{t} \mid I\right]$, where $I$ is the $(d-n) \times(d-n)$ identity matrix.

If $\mathcal{A}$ is an essential arrangement with matrix $A$, we define its (orthogonal) dual $\mathcal{A}^{\perp}$ to be the arrangement obtained from a dual of the matrix $A$. Note that because we discard zero columns and ignore multiplicities, $\mathcal{A}$ is not reconstructible from $\mathcal{A}^{\perp}$ in general.

We will also need several elementary facts from the theory of matroid duality (see $[3,4,10,17])$. There are many ways to define the orthogonal dual of a matroid. For us, the best definition is the circuit-cocircuit definition. Recall that a circuit is a minimal dependent set and a cocircuit is the complement of a copoint. The (orthogonal) dual $M^{\perp}$ of $M$ is the matroid on the same ground set whose circuits are exactly the cocircuits of $M$. Duality interchanges contraction and deletion, that is, for a subset $B$ of the set of elements, $\left(M^{\perp}\right) / B$ equals $(M \backslash B)^{\perp}$. It is true (but not obvious) that the matroid of the dual arrangement $\mathcal{A}^{\perp}$ is the simplification of the dual of the matroid of $\mathcal{A}$. Despite its age, the neatest and most accessible proof of this remains Whitney's original proof in [17].

A loop is an element $e$ such that the set $\{e\}$ is a circuit. An isthmus is an element $e$ such that $\{e\}$ is a cocircuit, so that $M$ is the direct $\operatorname{sum}(M \backslash\{e\}) \oplus\{e\}$. For graphs, an isthmus is an edge whose removal increases the number of connected components. Duality interchanges loops and isthmuses. 
We shall call closure in the dual matroid $M^{\perp} \perp$-closure.

Lemma 3.1. Let $M$ be a matroid on the set $E$ and $B \subseteq E$. Then $e$ is in the $\perp$-closure of $B$ if and only if $e$ is in $B$ or $e$ is an isthmus in the deletion $M \backslash B$. In particular, $a$ cocircuit $B$ is $\perp$-closed if its complementary copoint $X$ has no isthmuses.

Proof: The lemma follows from dualizing the statement: a point $e$ is in the $\perp$-closure if and only if $e$ is a loop in the contraction $M^{\perp} / B$.

The cycle matroid $M(G)$ of a graph is the matroid on the edge set whose circuits are the cycles of the graph. A cutset in a graph $G$ is an edge-subset whose removal increases the number of connected components of $G$. The circuits of the dual matroid $G^{\perp}$ are precisely the minimal cutsets of $G$. For graphs, an isthmus is an edge which is a cutset by itself. Thus, Lemma 3.1 gives an easy way to determine whether a minimal cutset is $\perp$-closed. We remark that the set of all edges incident to a vertex $v$ is a minimal cutset. Such "vertex cutsets" usually contain few edges compared to other minimal cutsets.

The complete graph $K_{n}$ is the graph on $n$ vertices with all possible edges. The maximum size of a minimal cutset in complete graphs is given in the next lemma.

Theorem 3.2. The largest cocircuit in the cycle matroid $M\left(K_{n+1}\right)$ has size $\lceil n(n+$ 2)/47. When $n \geq 5$, the largest cocircuits are $\perp$-closed.

Proof: Because $K_{n+1}$ contains all possible edges, minimal cutsets are in bijection with partitions of the vertex set into two non-empty subsets and these cutsets disconnect $K_{n+1}$ into two disjoint smaller complete graphs. By Lemma 3.1, every minimal cutset in $K_{n+1}$ gives a $\perp$-closed cocircuit with the exception of the cutsets which divide $K_{n+1}$ into a $K_{n-1}$ and a single edge $K_{2}$. The largest minimal cutsets are those which divide $K_{n+1}$ into two connected components of almost equal size. We conclude that the largest cocircuit in $M\left(K_{n+1}\right)$ has size $k^{2}$ if $n+1=2 k$ and $k(k+1)$ if $n+1=2 k+1$. To finish, it is easy to check that $\lceil n(n+2) / 4\rceil$ equals $k^{2}$ or $k(k+1)$ depending on the parity of $n+1$.

Theorem 3.3. Let $M$ be a rank-n matroid on the set $S$ with a modular copoint $X$. Suppose that the cocircuit $S \backslash X$ spans. If $n \geq 5$, then there exists $a \perp$-closed cocircuit in $M$ of size at least $\lceil n(n+2) / 4\rceil$.

Proof: We shall use the following lemma.

Lemma 3.4. Under the hypotheses in the theorem, M contains a spanning submatroid isomorphic to $M\left(K_{n+1}\right)$.

Proof: This is a combination of Lemma 5.3 in [7] and Lemma 5.14 in [8]. For the sake of completeness, we will give a proof in the language of arrangements and linear forms. Choose coordinates so that the linear forms $x_{i}, 1 \leq i \leq n$ are in the cocircuit $S \backslash X$ and the copoint $X$ is the subarrangement of all linear forms whose kernel contains 祭 Springer 
the point $(1,1, \ldots, 1)$. By modularity,

$$
\operatorname{rank}\left(\left(x_{i} \vee x_{j}\right) \wedge X\right)=\operatorname{rank}(X)+\operatorname{rank}\left(x_{i} \vee x_{j}\right)-n=1
$$

for every pair $x_{i}$ and $x_{j}$ of linear forms. Hence, there is a linear combination of $x_{i}$ and $x_{j}$ whose kernel contains $(1,1, \ldots, 1)$. This form is $x_{i}-x_{j}$, so the arrangement contains the subarrangement $\left\{x_{i}, x_{i}-x_{j} \mid 1 \leq i<j \leq n\right\}$, which is isomorphic to the graphic arrangement of $K_{n+1}$.

Let $K$ be a spanning submatroid in $M$ isomorphic to $M\left(K_{n+1}\right)$. Take a copoint $X^{\prime}$ in the submatroid $K$ and let $X$ be the closure of $X^{\prime}$ in $M$. A point in $K \backslash X^{\prime}$ is still not in $X$. Hence,

$$
|S \backslash X| \geq\left|K \backslash X^{\prime}\right|
$$

Choosing $X^{\prime}$ in $M \mid K$ so that $K \backslash X^{\prime}$ has size $\lceil n(n+2) / 4\rceil$, we obtain a cocircuit in $M$ having size at least $\lceil n(n+2) / 4\rceil$. If $n \geq 5$, the copoint $X^{\prime}$ in $K$ contains no isthmuses. Since $X$ and $X^{\prime}$ have the same rank, a direct summand of $X$ induces a direct summand of $X^{\prime}$. As $X^{\prime}$ contains no isthmuses, $X$ also contains no isthmuses and the cocircuit $S \backslash X$ is $\perp$-closed.

Corollary 2.3 and Theorem 3.3 imply Theorem 1.1 .

Since the matroid of the braid arrangement $A_{n}$ is $M\left(K_{n}\right)$, Theorem 2.2 and Theorem 3.2 imply that the projective dimension of the dual of the "essential" braid arrangement $\hat{A}_{n}$ is at least $\lceil(n-1)(n+1) / 4\rceil-3$. Lower bounds for the other families of real reflection arrangements can be obtained using the method in the proof of Theorem 3.2.

Theorem 3.5. When $n \geq 5$,

$$
\operatorname{pdim}\left(B_{n}^{\perp}\right) \geq\left\lfloor\frac{2}{3} n^{2}+\frac{1}{3} n-\frac{1}{24}\right\rfloor-3 .
$$

When $n \geq 6$,

$$
\operatorname{pdim}\left(D_{n}^{\perp}\right) \geq\left\lfloor\frac{2}{3} n^{2}-\frac{1}{3} n+\frac{1}{24}\right\rfloor-3
$$

Proof: Consider the copoint isomorphic to the direct sum $A_{k} \oplus B_{n-k}$ in $B_{n}$ spanned by the $n-1$ linear forms

$$
x_{1}-x_{2}, x_{2}-x_{3}, \ldots, x_{k-1}-x_{k}, x_{k+1}, x_{k+2}, \ldots, x_{n} .
$$

The cocircuit complementary to $X$ has size

$$
\left(\begin{array}{l}
k \\
2
\end{array}\right)+k+2 k(n-k) .
$$


We obtain a cocircuit of maximum size when $k$ is the integer closest to $2 n / 3+\frac{1}{6}$ and this maximum size is obtained by substitution into formula (1) and rounding down. Since $A_{2}$ and $B_{1}$ contain a single form, the cocircuits of maximum size are $\perp$-closed if $n \geq 5$. The argument for $D_{n}$ is similar.

The argument for $B_{n}$ can also be applied to the complex reflection arrangements $G(n, 1, l)$ to give a rough lower bound of $\left(l^{2} /(2 l+2)\right) n^{2}$ for $\operatorname{pdim}\left(G(n, 1, l)^{\perp}\right)$ when $n \geq 5$.

Acknowledgements The Macaulay2 software package, available at http: / www. math. uiuc. edu/ Macaulay / allowed us to compute many examples which provided evidence for the paper. In particular, Examples 2.5 and 2.6 were computed using this software. We thank two referees for useful comments.

\section{References}

1. K. Brandt and H. Terao, "Free arrangements and relation spaces," Discrete Comput. Geom. 12 (1994), 49-63.

2. A.B. Coble, "Associated sets of points," Trans. Amer. Math. Soc. 24 (1922), 1-20.

3. H.H. Crapo and G.-C. Rota, On the Foundations of Combinatorial Theory: Combinatorial Geometries. Preliminary edition, M.I.T. Press, Cambridge MA, 1970.

4. H.H. Crapo, "Orthogonality," in Theory of Matroids, N.L. White (Ed.), Cambridge Univ. Press, Cambridge, 1986, pp. 76-96.

5. I. Dolgachev and M. Kapranov, "Arrangements of hyperplanes and vector bundles on $P^{n}$," Duke Math. J. 71 (1993), 633-664.

6. D. Eisenbud, Commutative Algebra with a View Towards Algebraic Geometry. Graduate Texts in Mathematics, Vol. 150, Springer-Verlag, Berlin-Heidelberg-New York, 1995.

7. J.P.S. Kung, "Numerically regular hereditary classes of combinatorial geometries," Geom. Dedicata 21 (1986), 85-105.

8. J.P.S. Kung, "Critical problems," in Matroid Theory, J.E. Bonin, J.G. Oxley, and B. Servatius, (Eds.) Contemporary Math. Vol. 197, Amer. Math. Soc., Providence RI, 1996, pp. 1-127.

9. P. Orlik and H. Terao, "Arrangements of hyperplanes," Grundlehren Math. Wiss., Bd. 300, SpringerVerlag, Berlin-Heidelberg-New York, 1992.

10. J.G. Oxley, Matroid Theory, Oxford University Press, Oxford, 1992.

11. L. Rose and H. Terao, "A free resolution for the module of logarithmic forms of a generic arrangement," J. Algebra 136 (1991), 376-400.

12. R.P. Stanley, "Modular elements of geometric lattices," Algebra Universalis 1 (1971), 214-217.

13. R.P. Stanley, "Supersolvable lattices," Algebra Universalis 2 (1972), 197-217.

14. H. Terao, "Generalized exponents of a free arrangement of hyperplanes and Shepard-Todd-Brieskorn formula," Invent. Math. 63 (1981), 159-179.

15. H. Terao, "On the homological dimensions of arrangements," Unpublished manuscript, 1990.

16. H. Whitney, "Non-separable and planar graphs," Trans. Amer. Math. Soc. 34 (1932), 339-362.

17. H. Whitney, "On the abstract properties of linear dependence," Amer. J. Math. 57 (1935), 509-533.

18. M. Yoshinaga, "Characterization of a free arrangement and conjecture of Edelman and Reiner," Invent. Math. 157 (2004), 449-454.

19. S. Yuzvinsky, "A free resolution for the module of derivations for generic arrangements," J. Algebra 136 (1991), 432-438.

20. S. Yuzvinsky, "Cohomology of local sheaves on arrangement lattices," Proc. Amer. Math. Soc. 112 (1991), 1207-1217.

21. G. Ziegler, "Combinatorial construction of logarithmic differential forms," Adv. Math. 76 (1989), $116-154$. 